To appear in The Astrophysical Journal Letters

\title{
TIME VARIATION IN G24.78+0.08 A1: EVIDENCE FOR AN ACCRETING HYPERCOMPACT H II REGION?
}

\author{
Roberto Galván-Madrid ${ }^{1,2}$, Luis F. Rodríguez ${ }^{1}$, Paul T. P. Ho ${ }^{2,3}$, and Eric Keto ${ }^{2}$
}

\begin{abstract}
Over a timescale of a few years, an observed change in the optically thick radio continuum flux can indicate whether an unresolved H II region around a newly formed massive star is changing in size. In this Letter we report on a study of archival VLA observations of the hypercompact H II region G24.78+0.08 A1 that shows a decrease of $\sim 45 \%$ in the 6 -cm flux over a five year period. Such a decrease indicates a contraction of $\sim 25 \%$ in the ionized radius and could be caused by an increase in the ionized gas density if the size of the $\mathrm{H}$ II region is determined by a balance between photoionization and recombination. This finding is not compatible with continuous expansion of the $\mathrm{H}$ II region after the end of accretion onto the ionizing star, but is consistent with the hypothesis of gravitational trapping and ionized accretion flows if the mass-accretion rate is not steady.
\end{abstract}

Subject headings: H II regions - ISM: individual (G24.78+0.08) — stars: formation

\section{Introduction}

The formation of massive stars $\left(M>8 M_{\odot}\right)$ by accretion presents a number of theoretical difficulties; among them, that once a star attains a sufficient mass its surface temperature

\footnotetext{
${ }^{1}$ Centro de Radioastronomía y Astrofísica, UNAM, Morelia 58090, México; l.rodriguez@astrosmo.unam.mx

${ }^{2}$ Harvard-Smithsonian Center for Astrophysics, 60 Garden Street, Cambridge MA 02138, USA; rgalvan, eketo@cfa.harvard.edu

${ }^{3}$ Academia Sinica Institute of Astronomy and Astrophysics, Taipei, Taiwan; pho@asiaa.sinica.edu.tw
} 
is high enough to produce a small $\mathrm{H}$ II region (see the reviews on ultracompact and hypercompact H II regions by Churchwell 2002; Kurtz 2005; and Hoare et al. 2007). The thermal pressure differential between the hot $\left(\sim 10^{4} \mathrm{~K}\right)$ ionized gas and the cold $(\sim 100 \mathrm{~K})$ molecular gas can potentially reverse the accretion flow of molecular gas and prevent the star from ever reaching a higher mass. However, recent models that include the effects of gravity (e.g., Keto 2007) have shown that if the H II region is small enough (ionized diameter $\leq 1000 \mathrm{AU}$ for total stellar mass $\geq 350 M_{\odot}$ ), the gravitational attraction of the $\operatorname{star}(\mathrm{s})$ dominates the thermal pressure and the molecular accretion flow can cross the ionization boundary and proceed toward the star as an ionized accretion flow within the H II region. In this stage the H II region is said to be gravitationally trapped by the star.

Previous studies have demonstrated a number of observational techniques bearing on the evolution of small H II regions around newly formed stars. Accretion flows onto and through H II regions can be directly observed by mapping molecular and radio recombination lines (RRLs) at very high angular resolution (e.g., Sollins et al. 2005; Keto \& Wood 2006). If the $\mathrm{H}$ II region is too small to be spatially resolved, the frequency dependence of the velocities and widths of RRLs can be used to infer steep density gradients and supersonic velocities within the H II regions (Keto et al. 2007), as expected from the presence of ionized accretion flows or bipolar outflows. Radio continuum observations at very high angular resolution, made at two epochs some years apart, can also be used to directly observe changes in size of small H II regions (Franco-Hernandez \& Rodriguez 2004). Changes in size indicate whether the evolution of the H II region is consistent with pressure-driven expansion or gravitational trapping.

In this Letter we demonstrate yet another technique - how a comparison of radio continuum observations at different epochs can be used to infer size changes in H II regions even if the observations do not spatially resolve them. At optically thick frequencies the radio continuum flux depends to first approximation only on the size of an $\mathrm{H}$ II region and is independent of its internal density structure. Therefore, even if the size is not known, a change in the flux over time still indicates a change in the size of the $\mathrm{H}$ II region. This technique is particularly valuable because it can be used on the smallest and youngest H II regions, and because lower angular resolution radio continuum observations generally require less observing time than spectral line and high angular resolution observations.

For the study presented in this Letter we selected the hypercompact (HC) H II region G24.78+0.08 A1 which lies at the center of a massive molecular accretion flow (Beltrán et al. 2004, 2006) and also has multi-epoch $6 \mathrm{~cm}$ radio continuum observations in the VLA archive. G24.78+0.08 was detected in the centimetric (cm) continuum by Becker et al. (1994), and later resolved by Codella et al. (1997) into a compact (A) and an extended (B) component. 
A millimeter (mm) interferometric study (Beltrán et al. 2004) revealed the presence of two massive rotating toroids centered in respective dust cores (A1 and A2). The compact cm emission comes from the mm component A1 (hereafter G24 A1), and has recently been resolved by Beltrán et al. (2007). If G24 A1 is ionized by a single star, its spectral type should be earlier than O9 (Codella et al. 1997). Also, G24 A1 likely powers a massive CO outflow (Furuya et al. 2002).

The infalling and rotating molecular gas and bipolar outflow all suggest ongoing accretion. However, based on proper motions of $\mathrm{H}_{2} \mathrm{O}$ masers around the $\mathrm{H}$ II region, Beltrán et al. (2007) and Moscadelli et al. (2007) proposed that at the present time, the H II region is expanding into the accretion flow. The suggested timescale for the expansion is short enough that we should be able to detect a corresponding increase in the optically thick radio continuum flux within a few years.

\section{Observations}

We searched the VLA 1 archive for multi-epoch observations centered in the G24 A1 region at optically thick frequencies (below $23 \mathrm{GHz}$ for G24 A1). Since in the optically thick part of the spectrum the flux density of the source scales as the angular size squared, flux density variations corresponding to size variations can be detected even in observations of modest angular resolution. We chose three data sets of 6 -cm observations in the $\mathrm{C}$ configuration, two from 1984 and one from 1989 (see Table 1).

The observations were made in both circular polarizations with an effective bandwidth of $100 \mathrm{MHz}$. The amplitude scale was derived from observations of the absolute amplitude calibrator 3C286. This scale was transferred to the phase calibrator and then to the source. We estimate an error not greater than $10 \%$ for the flux densities of the sources.

We edited and calibrated each epoch separately following the standard VLA procedures using the reduction software AIPS. Precession to J2000 coordinates was performed running the task UVFIX in the $(u, v)$ data. After self-calibration, we made CLEANed images with uniform weighting and cutting the short spacings (up to $10 \mathrm{~K} \lambda$ ) to minimize the presence of extended emission at scales larger than $\sim 20^{\prime \prime}$.

Before subtraction, we made the images as similar as possible. We restored the CLEAN components with an identical Gaussian beam HPBW 4.'79 × 3.'38, PA=-15. We applied

\footnotetext{
${ }^{1}$ The National Radio Astronomy Observatory is operated by Associated Universities, Inc., under cooperative agreement with the National Science Foundation.
} 
primary beam corrections and aligned the maps. No significant differences were found in the difference image between the 1984 May 11 and 14 images, as expected for such a small time baseline. We therefore averaged the two 1984 epochs into a single data set. In subtracting the final maps we allowed the 1984 image to have small $\left(\simeq 0.1^{\prime \prime}\right)$ shifts in position as well as a scaling of $\simeq 10 \%$ in amplitude. This was done in order to minimize the rms residuals of the difference image in the region of interest. A similar procedure was used by Franco-Hernández \& Rodríguez (2004) to detect a variation in the lobes of the bipolar UC H II region NGC 7538 IRS1. The individual maps, as well as the final difference image between 1984 and 1989 are shown in Figure 1.

\section{Discussion}

\subsection{The Expected Variation Trend}

\subsubsection{If G24 A1 Is Expanding}

Beltrán et al. (2007) suggested that the $7 \mathrm{~mm}$ and $1.3 \mathrm{~cm}$ continuum morphologies are consistent with limb-brightening from a thin, ionized-shell structure. Based on $\mathrm{H}_{2} \mathrm{O}$ maser proper motions (see Fig. 3 of Beltrán et al. 2007, or Fig. 4 of Moscadelli et al. 2007) they also suggested an expansion speed of $\sim 40 \mathrm{~km} \mathrm{~s}^{-1}$. The increase in flux corresponding to the increase in size due to expansion ought to be detectable in a few years.

\subsubsection{If G24 A1 Is Accreting}

If G24 A1 is the ionized inner portion of the star forming accretion flow, the longterm growth of the $\mathrm{H}$ II region due to the increasing ionizing flux of the star should be imperceptible. However, the H II region could change in size over an observable timescale if the gas density in the accretion flow is time variable. Because the mass of ionized gas within the H II region is very small compared to the mass of the accretion flow, even a small change in the flow density could affect the size of the H II region.

For example, we can obtain a lower limit for the mass of G24 A1 assuming that it is spherical and homogeneous. From the equations of Mezger \& Henderson (1967) we have that:

$$
\left[\frac{M_{H I I}}{M_{\odot}}\right]=3.7 \times 10^{-5}\left[\frac{S_{\nu}}{\mathrm{mJy}}\right]^{0.5} \quad\left[\frac{T_{e}}{10^{4} \mathrm{~K}}\right]^{0.175} \times
$$




$$
\left[\frac{\nu}{4.9 \mathrm{GHz}}\right]^{0.05}\left[\frac{D}{\mathrm{kpc}}\right]^{2.5}\left[\frac{\theta_{s}}{\operatorname{arcsec}}\right]^{1.5}
$$

where the flux density $S_{\nu}$ has been measured at a frequency $\nu$ in which the $\mathrm{H}$ II region is optically thin, $T_{e}$ is the electron temperature, $D$ is the distance to the region, and $\theta_{s}$ is its FWHP. Taking $S_{\nu}=101 \mathrm{mJy}, D=7.7 \mathrm{kpc}$, and $\theta_{s}=0$ ". 17 at $7 \mathrm{~mm}$ (Beltrán et al. 2007), and assuming $T_{e}=10^{4} \mathrm{~K}$, the ionized mass in G24 A1 would be $\sim 5 \times 10^{-3} M_{\odot}$. A higher limit to the mass can be obtained considering that HC H II regions should have density gradients $\left(n \propto r^{\alpha}\right.$, with $\alpha=-1.5$ to -2.5$)$ rather than being homogeneous (Keto 2007). In this case we obtain ionized masses between $1 \times 10^{-2} M_{\odot}$ and $3 \times 10^{-2} M_{\odot}$, a factor of $2-6$ higher than under the assumption of homogeneity, but still very small when compared to that of the surrounding molecular material or the ionizing star itself.

\subsection{The Observed Variation}

Figure 1 shows the individual maps of the 1984 May $11+14$ (1984.36) and 1989 June 23 (1989.48) epochs, as well as the difference image. Sources A1 and B (the former labeled A by Codella et al. 1997) are unresolved $\left(\theta_{s} \leq 2^{\prime \prime}\right)$, and the total emission is dominated by component B. However, the flux decrease is centered at the position of A1. We performed Gaussian fits to both components using the task JMFIT in AIPS, and the results are summarized in Table 2. We have checked the reliability of the fits to the image by making direct fits to the $(u, v)$ data. The values obtained from both techniques are entirely consistent but suggest that the errors given by the tasks used in the image (JMFIT) and (u,v) (UVFIT) fittings are underestimated by a factor of 2 . The errors given in Table 2 have been corrected by this factor.

The decrease in the flux density of G24 A1 between 1989.48 and 1984.36 is $5.1 \pm 1.1$ mJy, or $45 \pm 10 \%$. Component B shows no evidence of time variability, as expected for a more evolved H II region not associated with signs of current star-forming activity such as $\mathrm{H}_{2} \mathrm{O}$ or $\mathrm{OH}$ masers (Codella et al. 1997). We set an upper limit of $2 \%$ to the circularlypolarized emission of the sources, which indicates that we are not dealing with variable gyrosynchrotron emission from an active stellar magnetosphere.

\subsection{Is G24 A1 Accreting?}

The $\sim 45 \%$ flux decrease (i.e., a contraction of $\sim 25 \%$ in the ionized radius) we have detected at $6 \mathrm{~cm}$ toward G24 A1 is not consistent with the hypothesis that this H II region 
is expanding rapidly into the molecular accretion flow. Assuming a radius $R \sim 500 \mathrm{AU}$ and

an expansion velocity $v \sim 40 \mathrm{~km} \mathrm{~s}^{-1}$ (Beltrán et al. 2007), a flux increase of $\sim 20 \%$ should have been observed between the compared epochs.

We attribute the contraction of the H II region to an increase in its density produced by the enhancement of accretion, either caused by an isotropic increment in the mass-accretion rate or by the sudden accretion of a localized clump in the neutral inflow (for example, clumps have been observed in the accretion flow onto the UC H II region G10.6-0.4; Sollins and Ho 2005; Keto \& Wood 2006). Our data do not allow us to distinguish among these two possibilities.

The additional mass required to increase the density can be estimated. The ionized radius $r_{\mathrm{s}}$ scales roughly with the density $n$ as $r_{\mathrm{s}} \propto n^{-2 / 3}$ (if the radius of the $\mathrm{H}$ II region is set by a balance between photoionization and recombination) and at optically thick frequencies the flux density scales as $S_{\nu} \propto r_{\mathrm{s}}^{2}$. The ionized mass within G24 A1 is $\sim 1 \times 10^{-2} M_{\odot}$ (\$3.1.2). Therefore, the sudden accretion of $\sim 5 \times 10^{-3} M_{\odot}$ into the $\mathrm{H}$ II region would suffice to explain the observed $45 \%$ flux decrease. The molecular accretion flow around this H II region has a mass of $\sim 130 M_{\odot}$ (Beltrán et al. 2004). Thus, the required variation, $50 \%$ of the mass of the H II region, is only $0.001 \%$ of the total mass of the accretion flow.

Finally, we shall mention that even when the observations here reported are not consistent with a simple, continuous expansion of G24 A1, they do not rule out other more complex, non-steady evolutionary scenarios.

\section{Conclusions}

Our analysis of archival VLA observations of the H II region G24.78+0.08 A1 indicates a contraction of its radius between 1984.36 and 1989.48. This finding is consistent with the hypothesis that this HC H II is the inner, ionized part of the larger scale accretion flow seen in the molecular line observations of Beltrán et al. (2004, 2006). Future high-resolution RRL observations could unambiguously resolve the ionized-gas kinematics and confirm this hypothesis.

\section{REFERENCES}

Becker, R. H., White, R. L., Helfand, D. J., \& Zoonematkermani, S. 1994, ApJ, 91, 347

Beltrán, M. T., Cesaroni, R., Codella, C., Testi, L., Furuya, R. S., \& Olmi, L. 2006, Nature, 
443,427

Beltrán, M. T., Cesaroni, R., Moscadelli, L., \& Codella C. 2007, A\&A, 471, L13

Beltrán, M. T., Cesaroni, R., Neri, R., Codella, C., Furuya, R. S., Testi, L., \& Olmi, L. 2004, ApJ, 601 L187

Codella, C., Testi, L., \& Cesaroni, R. 1997, A\&A, 325, 282

Churchwell, Ed. 2002, ARA\&A, 40, 27

Franco-Hernández, R., \& Rodríguez, L. F. 2004, ApJ, 604, L105

Furuya, R. S., Cesaroni, R., Codella, C., Testi, L., Bachiller, R., \& Tafalla, M. 2002, A\&A, 390, L1

Hoare, M. G., Kurtz, S. E., Lizano, S., Keto, E., \& Hofner, P. 2007, in Protostars and Planets V, ed. B. Reipurth, D. Jewitt, \& K. Keil (Tucson: Univ. Arizona Press), 181

Keto, E. 2007, ApJ, 666, 976

Keto, E., \& Wood, K. 2006, ApJ, 637, 850

Keto, E., Zhang, Q., \& Kurtz, S. 2007, preprint (arXiv:0708.3388)

Kurtz, S. 2005, in IAU Symp. 227, Massive Star Birth: A Crossroad of Astrophysics, ed. R. Cesaroni et al. (Cambridge: Cambridge Univ. Press), 111

Mezger, P. G., \& Henderson, A. P. 1967, ApJ, 147, 471

Moscadelli, L., Goddi, C., Cesaroni, R., Beltrán, M. T., \& Furuya, R. S. 2007, A\&A, 472, 867

Sollins, P. K., \& Ho, P. T. P. 2005, ApJ, 630, 987

Sollins, P. K., Zhang, Q., Keto, E., \& Ho, P. T. P. 2005, ApJ, 624, L49 
Table 1. Observational Parameters

\begin{tabular}{|c|c|c|c|c|c|c|}
\hline \multirow[t]{2}{*}{ Epoch } & \multicolumn{2}{|c|}{ Phase Center $^{\mathrm{a}}$} & \multirow{2}{*}{$\begin{array}{l}\text { Amplitude } \\
\text { Calibrator }\end{array}$} & \multirow{2}{*}{$\begin{array}{l}\text { Phase } \\
\text { Calibrator }\end{array}$} & \multirow{2}{*}{$\begin{array}{c}\text { Bootstrapped Flux } \\
\text { Density (Jy) }\end{array}$} & \multirow{2}{*}{$\begin{array}{c}\text { Beam } \\
(\operatorname{arcsec} \times \text { arcsec; deg })\end{array}$} \\
\hline & $\alpha(\mathrm{J} 2000)$ & $\delta(\mathrm{J} 2000)$ & & & & \\
\hline 1984 May 11 & 183612.145 & -071128.17 & $3 \mathrm{C} 286$ & $1743-038$ & $2.414 \pm 0.005$ & $5.38 \times 3.44 ;-29$ \\
\hline 1984 May 14 & 183612.145 & -071128.17 & $3 \mathrm{C} 286$ & $1743-038$ & $2.464 \pm 0.007$ & $4.78 \times 3.34 ;+03$ \\
\hline 1989 Jun 23 & 183610.682 & -071119.87 & $3 \mathrm{C} 286$ & $1834-126$ & $0.153 \pm 0.001$ & $4.50 \times 3.31 ;-10$ \\
\hline
\end{tabular}

${ }^{a}$ Units of right ascension are hours, minutes, and seconds. Units of declination are degrees, arcminutes, and arcseconds. 
Table 2. Gaussian Fit Results

\begin{tabular}{cccccc}
\hline \hline \multirow{2}{*}{ Epoch } & \multirow{2}{*}{ Component } & \multicolumn{3}{c}{ Position $^{\mathrm{a}, b}$} & \multirow{2}{*}{$\begin{array}{c}\text { Flux Density } \\
(\mathrm{mJy})\end{array}$} \\
\cline { 3 - 5 } & & & $\alpha(\mathrm{J} 2000)$ & $\delta(\mathrm{J} 2000)$ & \\
\hline 1984.36 & $\mathrm{~A} 1$ & 183612.545 & -071210.87 & $11.4 \pm 0.8$ \\
1984.36 & $\mathrm{~B}$ & 183612.668 & -071215.37 & $31.4 \pm 1.0$ \\
1989.48 & $\mathrm{~A} 1$ & 183612.545 & -071210.87 & $06.3 \pm 0.8$ \\
1989.48 & $\mathrm{~B}$ & 183612.668 & -071215.37 & $32.8 \pm 0.8$ \\
\hline
\end{tabular}

${ }^{a}$ Units of right ascension are hours, minutes, and seconds. Units of declination are degrees, arcminutes, and arcseconds.

${ }^{\mathrm{b}}$ For the 1989.48 epoch, the centers of the Gaussians were fixed to those obtained for the 1984.36 epoch. 

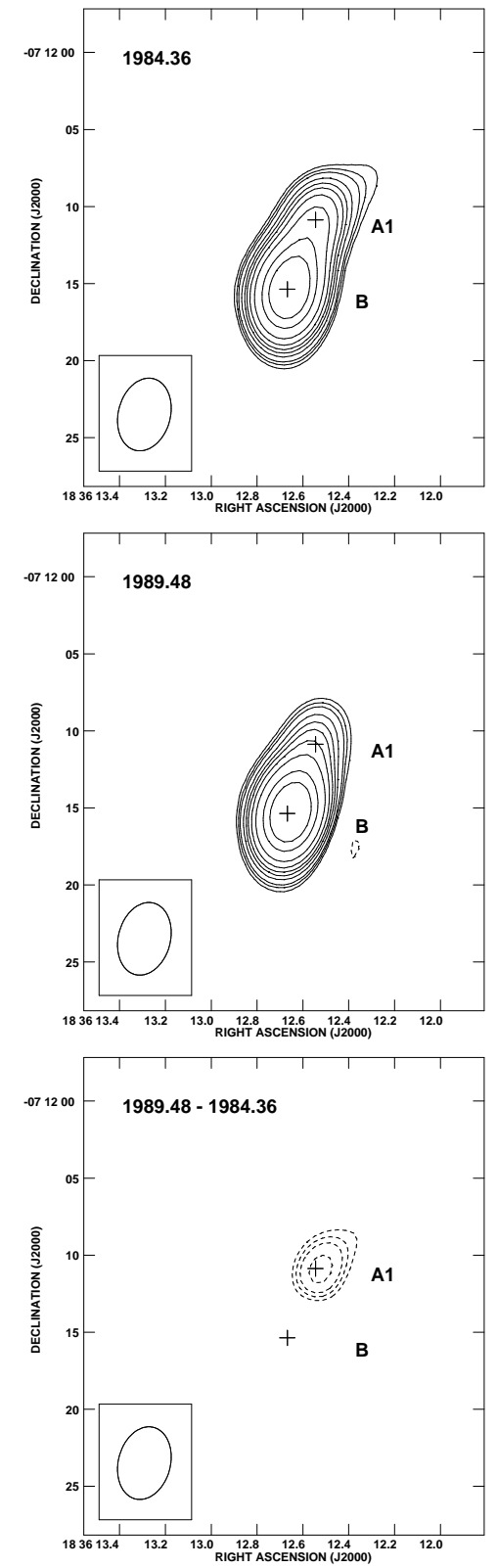

Fig. 1.- VLA images of G24.78+0.08 for 1984.36 (top), 1989.48 (middle), and the difference of 1989.48 - 1984.36 (bottom). The contours are -10, -8, -6, -5, -4, 4, 5, 6, 8, 10, 12, 15, 20, 30,40 , and 60 times $0.57 \mathrm{mJy}_{\text {beam }}{ }^{-1}$. The half power contour of the synthesized beam $\left(4^{\prime \prime} .79 \times 3^{\prime \prime} .38\right.$ with a position angle of $\left.-15^{\circ}\right)$ is shown in the bottom left corner of the images. The crosses indicate the positions of the components A1 and B from our Gaussian fits to the 1984.36 image. The negative residuals observed in the difference image indicate a decrease of $\sim 45 \%$ in the flux density of component A1. 